\title{
SORI Excitation: Collisional and Radiative Processes
}

\author{
Csaba Peltz, László Drahos, and Károly Vékey \\ Chemical Research Center, Hungarian Academy of Sciences, Budapest, Hungary
}

\begin{abstract}
Theoretical modeling of sustained off-resonance irradiation collision-induced dissociation (SORI-CID) experiments in Fourier transform ion cyclotron resonance (FT-ICR) mass spectrometry is described in the present paper. Manipulation of various analytical expressions yield the result that the average laboratory frame collision energy is equal to $2 / 3$ of the maximum kinetic energy in SORI. Survival yields (the fraction of nondecomposed molecular ions) as a function of excitation time, collision energy, and source temperature have been considered: results of MassKinetics-type reaction kinetics modeling were compared with experimental results obtained by Guo et al. (Int. J. Mass Spectrom. 2003, 225, 71-82). The results show that radiative cooling has a major influence in SORI-CID. They also suggest that collisional cooling is significant only at very low (less than $0.02 \mathrm{eV}$ ) center of mass collision energy; therefore it has a very small influence on the SORI process. Survival yield curves showed excellent agreement between experiments and calculations optimizing two parameters only (collisional energy-transfer efficiency and radiative cooling rate). Using leucine enkephalin as a model compound, the results indicate $0.128 \pm 0.021$ energy deposition in a single collision and $7.5 \pm 0.5 \mathrm{~s}^{-1}$ infrared cooling rate. We also present that these two physical parameters cannot be properly deconvoluted. This effect shows the importance of the parallel consideration of different physical processes. (J Am Soc Mass Spectrom 2007, 18, 2119-2126) (c) 2007 American Society for Mass Spectrometry
\end{abstract}

$\mathrm{I}$ on trapping techniques and tandem mass spectrometry became widely used in the past few decades for studying organic molecules. Paul's 3D trap and related instruments, such as linear traps and ion cyclotron resonance cells, became widespread in various fields of research [1-3]. Beside their recent application in proteomics [4], Fourier transform magnetic ion cyclotron resonance (FT-ICR) spectrometers have a dominant role in the field of gas-phase ion chemistry [5-9, 10]. Advantages of FT-ICR such as very high-resolution, flexibility of ion excitation techniques in the ICR cell [1, $11,12]$, and accurately controllable excitation conditions are important for ion chemistry studies.

Sustained off-resonance irradiation collision-induced dissociation (SORI-CID) is a widely used technique for dissociating molecules in FT-ICR $[13,14]$. The technique uses a periodical acceleration and deceleration of the ions combined with applying a relatively high, ca. $10^{-5}$ to $10^{-6}$ torr pressure collision gas to increase the internal energy. Note that the same pressure would be considered low using other techniques. Unlike onresonance excitation techniques $[15,16]$, SORI provides the capability of gradually depositing a large amount of internal energy in a controllable manner using a long activation time in combination with relatively low ki-

Address reprint requests to Dr. László Drahos, Chemical Research Center Hungarian Academy of Sciences, H-1025 Budapest, Pusztaszeri út 59-67, Hungary. E-mail: drahos@chemres.hu netic energies. In this sense it is similar to other slow heating methods [17], in particular to CID in 3D and linear ion traps [18]. SORI is well suited for consecutive tandem mass spectrometric measurements $\left(\mathrm{MS}^{n}\right)$ for structure elucidation and for ion chemistry studies and is also suitable for high mass molecules. SORI combined with resonance excitation offers particular advantages, as discussed recently [12].

Using advanced computation facilities, SORI has been modeled in different ways. SORI excitation is usually treated as a periodic acceleration and deceleration coupled to a number of random collisions. Statistical treatment of these collisions gave birth to MonteCarlo type models. Fujiwara et al. estimated probability density functions of ion-molecule collisions with several types of pseudo-random number generators [19]. Other papers use the Rice-Ramsperger-Kassel-Marcus / quasi-equilibrium theory (RRKM/QET) [12, 20, 21]. Some of these studies point out the importance of the pressure of collision gas, which is related to the number of collisions during a SORI cycle [20, 22, 23].

Another effect to consider is radiative cooling/heating of ions in a mass spectrometer [10, 24, 25]. To be significant, radiative processes require long residence times, therefore are often neglected in mass spectrometry. However, in FT-ICR, due to the long residence times used, they are significant and should be considered. At temperatures/internal energies typically observed in mass spectrometry (especially after excita- 
tion), radiative processes occur predominantly at infrared frequencies and mostly radiative cooling is observed. However, the importance of radiative processes may be best illustrated by excitation, e.g., the blackbody infrared irradiation dissociation (BIRD) technique [26], where excitation occurs by radiative heating.

Probably the first attempt to measure collisional energy-transfer in FT-ICR was performed using onresonance CID. It was determined that in a single collision, $9.6 \%$ of the center of mass (com) collisional energy $\left(E_{\text {com }}\right)$ was converted to internal energy [8]. This is commonly called collisional energy-transfer efficiency $\left(\eta\right.$, or more precisely $\left.\eta_{\text {com,single }}\right)$, but terms like energy conversion efficiency or $\mathrm{T}$ to $\mathrm{V}$ energy-transfer is also used. Note that the maximum amount of kinetic energy which may be converted into internal energy in a single collision is equal to $E_{\text {com }}$ (i.e., $\eta=100 \%$ ). When there are many collisions, as is common in various experiments, the internal energy of the ion may be raised by much more than $E_{\text {com }}$, especially if the ions are continuously being accelerated, like in SORI. In the study quoted [8], infrared cooling was not considered, so the true energy-transfer efficiency may be larger than $9.6 \%$. Using a similar evaluation method, Guo et al. determined energy deposition (i.e., the increase of the internal energy of a selected ion) in the case of onresonance excitation [9] and also in SORI [10]. In these cases temperature-dependent experiments were performed, noting the importance of radiative cooling.

The methods discussed above evaluate survival yield (SY) curves, i.e., the proportion of not-dissociated molecular ions as a function of collision energy or collision time. Data evaluation is relatively simple, use only the 50\% survival yield (SY) point of the curves. While this method is sound and is very useful [8-10], its main drawback is that the shape of the SY curves is not taken into account. In the present paper, we use a more complex data evaluation technique, using the shape of SY curves as well (i.e., relying on all data points). SORI excitation will be modeled, considering the simultaneous effect of collisions, radiative cooling, and fragmentation. Two parameters will be determined, $\eta_{\text {com,single }}$ and $k_{\text {cool }}$, characterizing collisional energytransfer efficiency and the radiative cooling rate, respectively. All other parameters needed for modeling were taken from the literature $[9,10]$.

\section{Experimental and Computational Details}

The modeled temperature-dependent SORI and onresonance experiments were carried out by Guo et al. on a modified Bruker APEX 7.0e FT-ICR mass spectrometer (Bremen, Germany) [10, 27]. Leucine enkephalin (YGGFL) was studied using argon collision gas. The protonated molecule $(\mathrm{m} / \mathrm{z} 556)$ of leucine enkephalin was selected and isolated, ejecting all other ions. After a few seconds of thermalization delay SORI experiments were carried out. RF off-resonance excitation was applied at $\Delta \nu=-1000 \mathrm{~Hz}$ with excitation amplitudes $V_{p-p}$
$=2.25,2.75,3.00$ and $3.25 \mathrm{~V}$. These amplitudes correspond to the maximum laboratory-frame collisional kinetic energies of 3.9, 5.9, 7.0 and 8.2 eV. The measurements were repeated at different cell temperatures in the 143 to $296 \mathrm{~K}$ range. The ion survival yield versus the SORI-CID activation time, i.e., the number of the SORICID cycles was registered. Argon was used as collision gas $\left(5 \mathrm{~s}\right.$, peak pressure $\left.=5 \times 10^{-6} \mathrm{mbar}\right)$ throughout the study. Further experimental details are described in Guo's paper [10].

The results of these experiments-survival yield as a function of time, temperature, and kinetic energywere examined using the MassKinetics model [28, 29]. The algorithm of MassKinetics deals with all the important physical processes influencing the product distribution in a mass spectrometer. Reactions, effects of acceleration, photon exchange, and collisional processes are simultaneously considered using the master equation approach. The studied molecular system is described by the relative abundance of reactants and products, and by the respective kinetic and internal energy distribution for each ion. Time development of these distributions is calculated using the master equation approach, beginning with ion formation-in this case from the isolation of the molecular ion-to the detection of the product ions. The result of the calculation is a product distribution, corresponding to the observed mass spectrum. Internal energy dependent reaction rates are estimated by the transition-state theory (RRKM formalism). Further details of the theoretical model and MassKinetics model can be found elsewhere $[9,28]$.

Fragmentation and collisional excitation of leucineenkephalin was studied using argon collision gas, the pressure of argon was $5 \times 10^{-6}$ mbar, as mentioned above. Note that these experimental conditions, as usual in SORI-CID, represent the low-pressure limit, i.e., less then one collision/RF cycle. The survival yield of the molecular ion was calculated using the approach of MassKinetics described previously $[9,28]$. The molecular parameters needed for the calculations were taken from previous studies; the critical energy and pre-exponential factor from the work of Williams and coworkers [30]. Vibrational frequencies for leucine enkephalin were taken from our previous work [12], where it has been determined by quantum chemical calculations using the density functional theory at the B3LYP level with 6-31G*(d) [31-33] basis function set. Further parameters needed for MassKinetics calculations are the mean collisional energy-transfer efficiency $(\eta)$ and the radiative cooling rate constant, which were taken as variables, and optimized during the calculations, as described later. An important aspect of collisional activation is not only the mean value, but the distribution of collisional energy-transfer, which has been studied in detail. Three distributions have been compared and will be discussed, that of Armentrout (determined experimentally) [39], Hase (determined using trajectory calculations [34], and a simple exponen- 
tial distribution, which is used most commonly. The collision model assumes hard-sphere collisions and that collisional energy-transfer is directly proportional to collision energy [34-37].

Radiative cooling is considered as described in MassKinetics [28], based on a revised version of Dunbar's original model [25], using the following equation:

$$
E_{\text {excess }}(t)=E_{\text {excess }}(0) \times \exp \left(-k_{\text {cool }} t\right)
$$

where $E_{\text {excess }}$ is the excess energy of the ions, $k_{\text {cool }}$ is an empirical constant describing the radiative decay rate, and $t$ is the time. The excess energy here is defined as the difference of $E_{\text {int }}$ (internal energy) and the mean thermal energy of the compound at the temperature of the environment, in the present case that of the mass spectrometer ion source. (Note that it is different from the definition of excess energy used in relation to reaction kinetics.)

$$
E_{\text {excess }}=E_{\text {int }}-E_{\text {therm, } \mathrm{MS}}
$$

Equation 1 indicates that if an ion is highly excited, like in various mass spectrometric experiments, it will emit photons to approach equilibrium with ion source temperature. The amount of radiative cooling depends on the degree of excitation: highly excited ions will loose energy more energy per unit time than ions which are only slightly excited. The value of $k_{\text {cool }}$ depends on the compound studied, but it is independent on molecular size and compounds of similar structure are characterized by similar $k_{\mathrm{cool}}$ values [25]. The same equation (and the same $k_{\text {cool }}$ value) describes radiative heating as well: if the ion (molecule) is "cold", it will be absorbing photons to get in equilibrium with the hot walls of the instrument (like in BIRD experiments). In such a case $\mathrm{E}_{\text {excess }}$ is a negative value and the $k_{\text {cool }}$ parameter will describe the rate of energy gain.

Applying the formalisms described above, product abundances were calculated by MassKinetics at the various experimental conditions, resulting in calculated survival yields. The rate constant of radiative cooling $\left(k_{\text {cool }}\right)$ and the collisional energy-transfer $\left(\eta_{\text {com,single }}\right)$ were fitted simultaneously on the whole dataset using different fitting algorithms like Powell's multi-dimensional direction set method and simulated annealing (for more details see numerical recipes on-line book, http://www.nr.com/). Note that collisional energytransfer always has a distribution, the efficiency $\left(\eta_{\text {com,single }}\right)$ being its mean value. When not mentioned otherwise, the distribution given by Hase [34] has been used in our calculations.

\section{Results and Discussion}

\section{Theoretical Modeling of the SORI Excitation Process}

In SORI experiments the velocity and therefore the (laboratory frame) kinetic energy $\left(E^{\mathrm{kin}}\right)$ of ions changes periodically, as shown graphically in Figure 1a and b. For a cylindrical ICR cell the time dependence of the kinetic energy is of the ions is described by eq 3 :

$$
E^{\mathrm{kin}}(t)=\frac{\beta^{2} q^{2} V_{p-p}^{2}}{64 \pi^{2} m d^{2} \Delta v^{2}}(1-\cos 2 \pi \Delta v t)
$$

where $\beta$ is the geometrical factor of the ICR cell, $q$ and $m$ are the charge and the mass of the ion respectively, $V_{p-p}$ is the peak-to-peak excitation voltage, $d$ is the diameter of the ICR cell, and $\Delta \nu$ is the difference between the cyclotron and RF excitation frequencies. The maximum kinetic energy during SORI experiments can be given therefore as:

$$
E_{\max }^{\mathrm{kin}}=\frac{\beta^{2} q^{2} V_{p-p}^{2}}{32 \pi^{2} m d^{2} \Delta v^{2}}
$$

Typically this $E_{\max }^{\mathrm{kin}}$ value is used in the literature $[20,23]$ as an indicator of kinetic energy during SORI experiments. Note that it is a maximum value, so that most of the time the ions will have smaller kinetic energy, as shown in Figure 1b.

The probability of collisions is also important in SORI, and it is given for an ion of $E^{\text {kin }}$ kinetic energy by:

$$
P^{\mathrm{coll}}\left(E^{\mathrm{kin}}\right)=\sqrt{E^{\mathrm{kin}}} / N
$$

where $\mathrm{N}$ is a normalization factor to provide an appropriate likelihood distribution function. Equation 5 assumes hard-sphere collisions. Because the collision cross-section for hard-spheres is velocity independent, it and the collision gas pressure are lumped together in the normalization factor. Furthermore, collisional energy-transfer (at least at low kinetic energy and in a small energy range) is assumed to be directly proportional to collision energy. Fast ions collide more frequently than

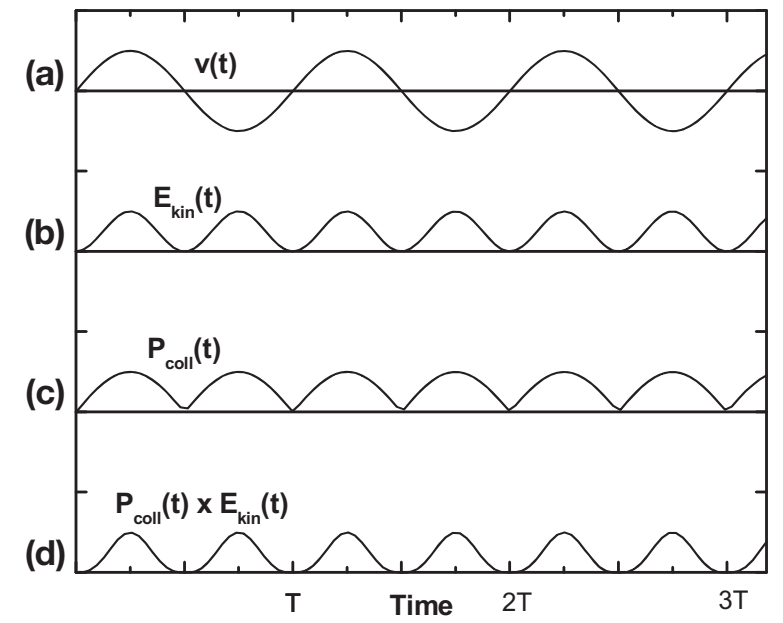

Figure 1. Velocity (a), kinetic energy (b), collision probability (c), and the product of collision probability and kinetic energy (d) as a function of time during a SORI-CID experiment. 
slow ones, therefore under SORI conditions the collision probability changes periodically in time, together with the kinetic energy. Using eqs 3 and 5, time dependence of the collision probability can be determined. This is described by eq 6 and is shown in Figure 1c.

$$
P^{\mathrm{coll}}(t)=\sqrt{\frac{\beta^{2} q^{2} V_{p-p}^{2}}{64 \pi^{2} m d^{2} \Delta v^{2}}(1-\cos 2 \pi \Delta v t) / N}
$$

Energy deposition in the molecule depends both on the collision energy and the collision probability, and will be approximately proportional to the product $E^{\mathrm{kin}}(t)$ * $P^{\text {coll }}(t)$; this is shown in Figure 1d.

Describing and modeling time-dependent processes is both difficult and time-consuming, so it is worth considering an alternative approach. The time dependence of the kinetic energy described by eq 3 was converted to a kinetic energy distribution. Assuming a uniform sampling over time, the probability of a given kinetic energy can be determined in from the timedependence of kinetic energy. This conversion (i.e., that of a uniform distribution to a distribution over a dependent parameter) can be calculated using an inverse function. This method can only be used for monotonic functions; so for periodic functions like the case of SORI, the energy-time function has been split to monotonic parts, and distributions obtained over these intervals were summed up afterwards. This method results in a kinetic energy distribution; described by eq 7 and shown in Figure 2a, corresponding to the kinetic energy sampled over several SORI cycles:

$$
P\left(E^{\mathrm{kin}}\right)=\frac{1}{\pi} \frac{E_{\max }^{\mathrm{kin}}}{\sqrt{\left(E_{\max }^{\mathrm{kin}}-E^{\mathrm{kin}}\right) E^{\mathrm{kin}}}}
$$

Also note that eq 7 is normalized to give unit integral on the $\left(0, E_{\max }^{\mathrm{kin}}\right)$ range. Figure $2 \mathrm{a}$ shows two maxima, indicating that most ions (or more accurately expressed, ions for most of the time) are either very slow or very fast, only few have medium kinetic energy. The kinetic energy distribution of those ions can also be determined, which do collide: this will be called "collision energy distribution". It is expressed by eq 8, and is shown in Figure 2b.

$$
\begin{aligned}
& P^{\mathrm{coll}}\left(E^{\mathrm{kin}}\right)=\frac{\sqrt{E^{\mathrm{kin}}} P\left(E^{\mathrm{kin}}\right)}{N} \\
& =\frac{\sqrt{E^{\mathrm{kin}}}}{N} \frac{1}{\pi} \frac{E_{\max }^{\mathrm{kin}}}{\sqrt{\left(E_{\max }^{\mathrm{kin}}-E^{\mathrm{kin}}\right) E^{\mathrm{kin}}}}
\end{aligned}
$$

$N$ being a normalization factor; note that the kinetic and collision energy distributions are characteristically different: slow ions practically do not collide, so there is no peak at low collision energy in Figure $2 b$. The expected value of time averaged (laboratory frame) collision energy $\left(E_{\text {lab,avg }}^{\text {coll }}\right)$ can be determined from eq 8 , and is given by eq 9:
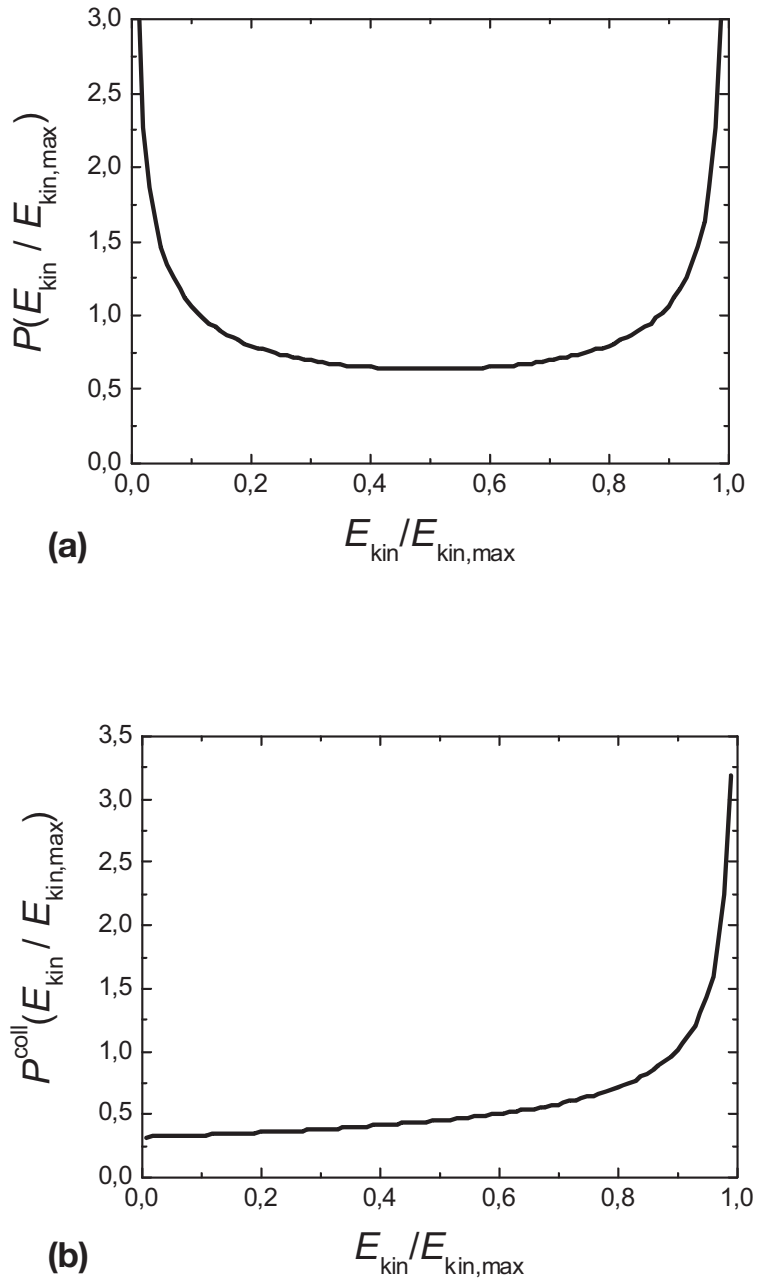

Figure 2. Time-averaged kinetic energy (a) and collision energy (b) distributions

$$
E_{\text {lab,avg }}^{\text {coll }}=\int_{0}^{\infty} E^{\mathrm{kin}} P^{\text {coll }}\left(E^{\mathrm{kin}}\right) d E^{\mathrm{kin}}=\frac{2}{3} E_{\max }^{\mathrm{kin}}
$$

This means that the mean collision energy in SORI can be very simply expressed as $2 / 3 E_{\max }^{\mathrm{kin}}$, indicating that the outcome of a SORI experiment would be identical to having all ions at a constant $2 / 3 E_{\max }^{\mathrm{kin}}$ kinetic energy. Energy-transfer in a collision $\left(\Delta E_{\text {int }}\right)$ can be conveniently expressed as a function of center-of-mass collision en$\operatorname{ergy}\left(E_{\mathrm{com}}\right)$ :

$$
\begin{aligned}
& \Delta E_{\text {int }}=\eta_{\text {single,com }} E_{\text {com }} \\
& E_{\text {com }}=E_{\max }^{\mathrm{kin}} \frac{m_{g}}{m_{g}+m}
\end{aligned}
$$

where $m_{\mathrm{g}}$ is the mass of collision gas, $m$ is the mass of the ion, $\eta_{\text {single,com }}$ is the energy-transfer efficiency. These equations drastically simplify SORI modeling, as changes in the kinetic energy do not have to be taken into account. This result also indicates that the conven- 
tional use of $E_{\max }^{\mathrm{kin}}$ for characterizing the SORI-CID [13, 38] is sound, characterizes collision energetics quite well.

\section{Numerical Modeling of SORI Experiments}

The results discussed above were incorporated into the framework of MassKinetics and used for numerical modeling of leucine enkephalin fragmentation under SORI conditions. Experimental data were taken from [10], the most important aspects of modeling were described above. Altogether 122 survival yield values have been determined experimentally varying the collision energy (characterized by $E_{\max }^{\mathrm{kin}}$ ), source temperature, and SORI excitation time. These data points have been calculated by modeling as well, using various combinations of $\eta_{\text {single,com }}$ and $k_{\text {cool }}$. Simultaneous optimization of these two parameters was performed to obtain best agreement between the experimental and theoretically calculated SY values.

First, modeling was performed without taking into account radiative cooling. The results are best viewed as a series of SY curves as a function of excitation time, obtained at different source temperatures and collision energies, and are shown in Figure 3 (experimental values [10] are shown by marks, theoretical values by continuous lines). The optimized value of $\eta_{\text {single,com }}$ is 0.066 (i.e., $6.6 \%$ ), but the agreement between the experimental and calculated curves is not good at all. All calculated curves are far steeper than the experimental ones, and the distance between the theoretical curves is much smaller than that observed experimentally. For example, Figure $3 \mathrm{~b}$ shows SY curves obtained at three different temperatures using the same collision energy. Difference between the three calculated curves is due to the difference in initial internal (thermal) energy; but this is clearly insufficient to describe the differences observed. Note that the amount of thermal energy can be calculated quite accurately and incomplete thermalization of the ions in the source would bring the experimental data closer and not farther from each other.

Prompted by one of the reviewers, we have examined the influence of the shape of collisional energytransfer on the results. Three different distributions have been compared; the mean of the distributions were characterized by the $\eta$ value (i.e., on average, $\eta$ fraction of $E_{\text {com }}$ is transferred to internal energy, but in a given collision energy-transfer may be anything between zero and $\left.\mathrm{E}_{\mathrm{com}}\right)$. It was also checked what happens when not a distribution but a single value is used for energytransfer. Optimization of $\eta$ has been repeated for all 122 data points as described above, but for simplicity only those relating to one experiment $\left(296 \mathrm{~K}, 7.0 \mathrm{eV} \mathrm{E}_{\mathrm{kin} \text {,max }}\right)$ is shown in Figure 3c. Triangles show the experimental results (same as those in Figure 3b); the dashed line is obtained using a simple exponential decay (which is probably the most commonly assumed distribution), while the dotted line is obtained using a single value for
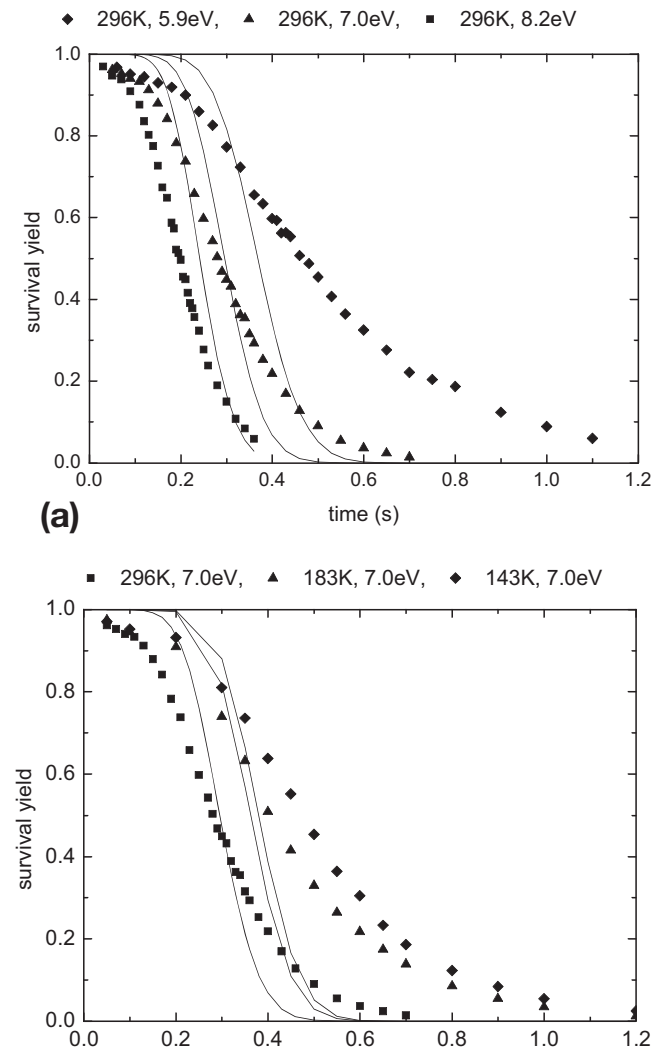

(b)

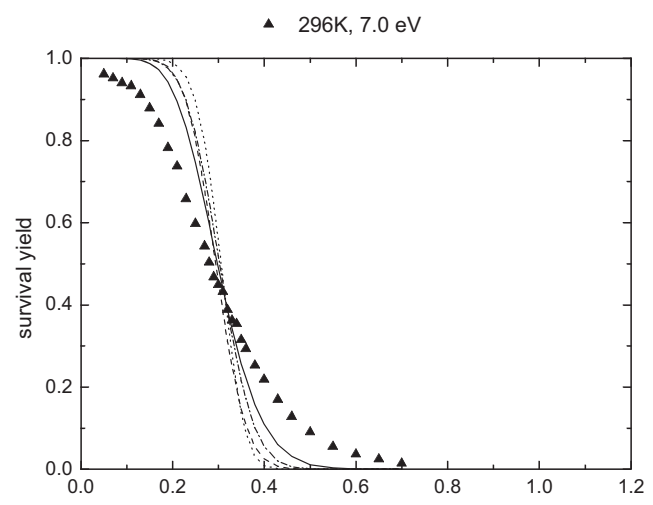

(c)

Figure 3. Experimental and calculated survival yield curves without taking into account collisional cooling. Solid lines show the results of modeling, mark the experimental values. (a) SY curves obtained at $296 \mathrm{~K}$ temperature at three different collision energies. (b) SY curves obtained at $7.0 \mathrm{eV}$ collision energy $\left(E_{\max }^{\mathrm{kin}}\right)$ at three different temperatures. (c) SY curves obtained at $296 \mathrm{~K}$ temperature and $7.0 \mathrm{eV}$ collision energy using different collisional energy-transfer distribution functions in calculations: solid line Hase's [34], dashed line exponential, dash-dot Armentrout's [39] CET function, and dotted line for single value energy-transfer.

energy-transfer. Recently, two energy-transfer distributions have been published: one by Hase (obtained using trajectory calculations on a small peptide, solid line in Figure 3c, [34] and another one by Armentrout (obtained experimentally on $\mathrm{Cr}(\mathrm{CO})_{6}{ }^{+}$molecules, dash-dot line in Figure 3c, [39]. The shapes of these two energytransfer distributions are quite similar (showing the 
influence of so-called "super-collisions"), and thought to be the best approximations currently available. The survival yield curves calculated by these two distributions are shown by solid and dash-dot lines in Figure 3c.

The results show that using a single value energytransfer yields the worst result (is farthest from the experimental values). Results using Hase's and Armentrout's distributions are similar, but neither these nor the exponential distribution are capable of explaining the low slope and long high-energy tail of the experimentally obtained SY curve. Note that the mean collisional energy-transfer $(\eta)$ obtained by optimization is very similar in all four cases: 0.065, 0.067, 0.066, and 0.065 (for single, exponential, Hase and Armentrout, respectively). Summarizing, the results do depend on the shape of collisional energy-transfer, but the differences do not appear to be very significant.

The model was than extended including radiative cooling, as described above. The results are shown in Figure 4, which shows a very satisfactory agreement between experimental and theoretically calculated SY values (marks and lines in Figure 4, respectively). This is especially pleasing after the fiasco of Figure 3. Note that only two parameters were optimized to fit 122 data points; all other aspects of modeling have been kept constant and were the same as in previous applications of MassKinetics [9, 12, 28, 40-42]. These increase confidence that the model developed is sound and describes the important aspects of fragmentation, excitation, and de-excitation of the studied system quite well.

The good agreement between the shapes of experimental and calculated SY curves (Figure 4a and b) show that the time-dependence of fragmentation (effect of the duration of the SORI pulse) is well described by the model. Careful study of the experimental data shows some deviation at the start of the SY curves: some fragmentation occurs at very short excitation time or even when a SORI pulse is not used at all. Most likely it is an experimental artifact, due to excitation/fragmentation during ion selection and/or detection, which is often observed in FT-ICR. After a few SORI cycles the calculated curves begin to fit better. Changes in the SY curves due to varying the collision energy (Figure 4a) and the source temperature (Figure $4 \mathrm{~b}$ ) are very well reproduced by the calculations.

Simultaneous fitting of the two parameters, mean collisional energy-transfer efficiency and radiative cooling rate, results in the optimized values of $\eta_{\text {single,com }}=$ 0.128 and $k_{\mathrm{cool}}=7.5 \mathrm{~s}^{-1}$, which is one of the main result of the present study. Note that collisional "heating" and radiative cooling has an opposite effect. When the latter is neglected, collisional energy-transfer efficiency appears to be much smaller. The result determined here compares well with the efficiency determined before for on-resonance excitation, without taking into account radiative cooling [8]. In the case of on-resonance excitation the influence of radiative cooling is smaller (due to the shorter time-scale of the collision cascade), so the 0.096 determined in that study [8] lies conveniently

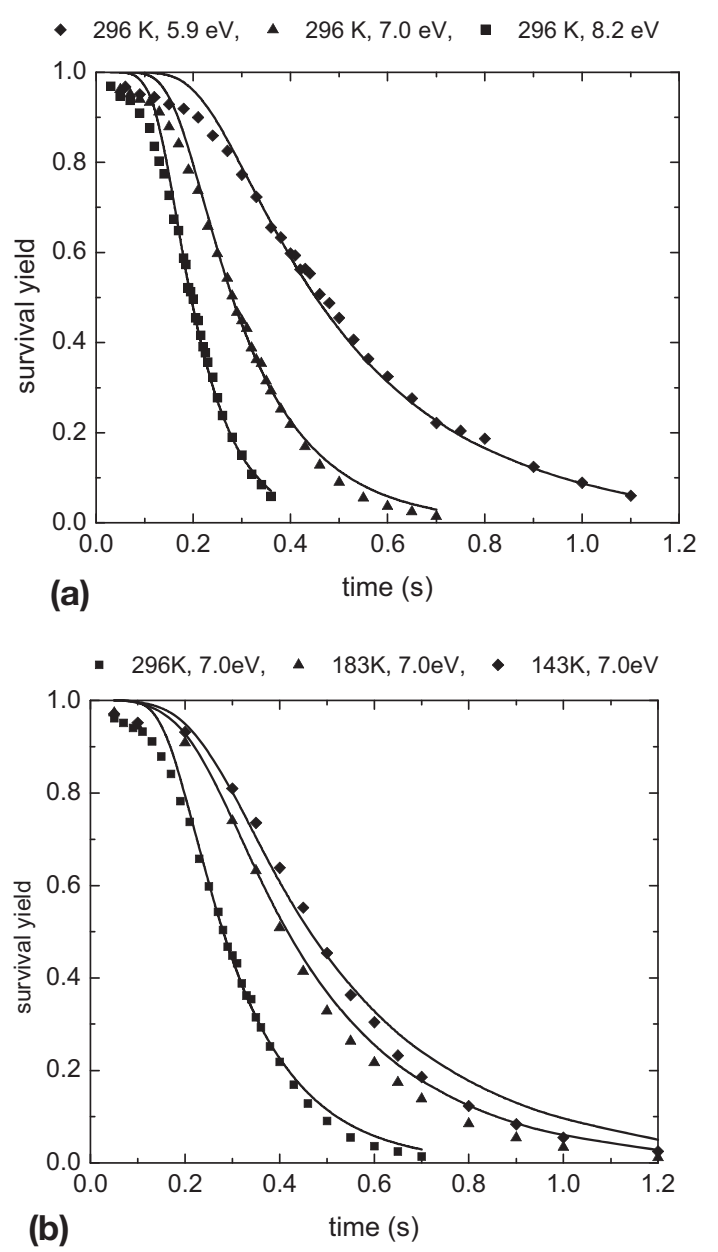

Figure 4. Experimental and calculated survival yield curves when collisional cooling is taken into account. Solid lines show the results of modeling, marks the experimental values. (a) SY curves obtained at $296 \mathrm{~K}$ temperature at three different collision energies. (b) SY curves obtained at $7.0 \mathrm{eV}$ collision energy $\left(E_{\max }^{\mathrm{kin}}\right)$ at three different temperatures. Results of the fitting procedure on the whole temperature-dependent dataset. Solid line shows the result of modeling, markers show the experimental values using (a) different initial kinetic energies, (b) different temperatures.

between the 0.066 (SORI, cooling not included) and 0.128 (SORI, cooling included).

Another phenomenon to consider is collisional cooling. This is significant only when there are a large number of collisions under thermal kinetic energies. In SORI ions are accelerated, so kinetic energies are much higher than that in a thermal system. To give a numerical estimate, $E_{\max }^{\mathrm{kin}}$ in the present case is in the order of around $7 \mathrm{eV}$, corresponding to $\mathrm{E}_{\mathrm{com}}$ of ca. $0.5 \mathrm{eV}$; typical for SORI conditions. In the SORI process leucine enkephalin does get excited; its mean internal energy increases approximately up to $3 \mathrm{eV}$ [12]. Approximately, this corresponds to leucine enkephalin heated up to $500 \mathrm{~K}$; at which temperature the thermal collision energy is $0.02 \mathrm{eV}$. Evaluating these, cooling collisions occur when the collision energy is less than $0.02 \mathrm{eV}$. This is only $4 \%$ that of the maximum collision energy used in SORI, and Figure $2 \mathrm{~b}$ suggests that only a small 
fraction, ca. $1 \%$ to $2 \%$ of all collisions are in this energy range. These suggest that collisional cooling has a small influence on SORI-CID, and may influence collisional energy-transfer processes only by a few \%. Accurate numerical calculations taking into account collisional cooling are outside the scope of the present paper.

Accuracy and reliability of the results is difficult to estimate. The size of errors in the modeling has been estimated by assuming reasonable errors in the initial parameters used in the modeling (those taken from [9] and [10]: $\pm 0.05 \mathrm{eV}$ error of critical energy, $\pm 10 \mathrm{~cm}-1$ of vibrational frequencies, $\pm 10 \%$ of pressure, and $\pm 1 \mathrm{~K}$ of temperatures measured, and furthermore using three different collisional energy-transfer curves), using random combinations of these errors, and checking the influence of these on $\eta_{\text {single,com }}$ and $k_{\text {cool }}$. The results show that errors (standard deviation) in $\eta_{\text {single,com }}$ are $0.128 \pm 0.021$, while errors in $k_{\text {cool }}$ are $\sim 7.5 \pm 0.5 \mathrm{~s}^{-1}$. Note that results obtained using Armentrout's collisional energy-transfer function [39] lies well within these error limits.

We believe interdependence of heating and cooling has a much larger influence on the results than that above, as underestimation of $\eta_{\text {single,com }}$ can easily be compensated by underestimating $k_{\text {cool }}$ or vice versa. To explore the significance of this phenomenon, accuracy of the fitting was studied as a function of energytransfer efficiency and radiative cooling rate constant. The fit of calculations (average distance between experimental and calculated points) on the experimental dataset was determined using several hundred points, and the results are shown as a 3D plot in Figure 5. The map shows a long, curving valley, indicating that several combinations of $\eta$ and $\ln \left(k_{\text {cool }}\right)$ values will give reasonably good fits with the experimental results.

\section{Conclusions}

Analysis of the time-dependence of kinetic energy changes during SORI excitation has been performed. Analytical manipulation of the various equations results in a simple expression: the mean laboratory-frame collision energy is equal to $2 / 3$ of the maximum kinetic energy in SORI-CID. This suggests that the maximum kinetic energy, often used to characterize the SORI process, is indeed a sound description of the collision process.

Experimentally obtained [10] survival yield (SY) curves as a function of excitation time, collision energy, and ion source temperature were compared with results of MassKinetics (reaction kinetics) modeling. Parameters of the theoretical model were taken from previous studies [9, 28]. Neglecting radiative cooling but optimizing collisional energy-transfer efficiency $\left(\eta_{\text {single,com }}\right)$, the model was unable to describe the shape of, or the distance between the SY curves (Figure 3). Fiddling with various other parameters of the MassKinetics model (transition-state, frequencies, activation energy, shape of the collision energy-transfer distribution, pressure in the ion source, etc.) was also useless. On the other hand, when radiative cooling was included, experiments and calculations started to fit together quite well. Using the original model $[9,28]$ and simultaneously optimizing collisional energy-transfer efficiency $\left(\eta_{\text {single,com }}\right)$ and radiative cooling rate $k_{\text {cool }}$, an excellent agreement between experiments and calculations were obtained, shown in Figure 4. Good agreement between the experimentally found and theoretically calculated SY curve shapes, the well-described distance between SY curves obtained at different temperatures and collision energies, and the fact that 122

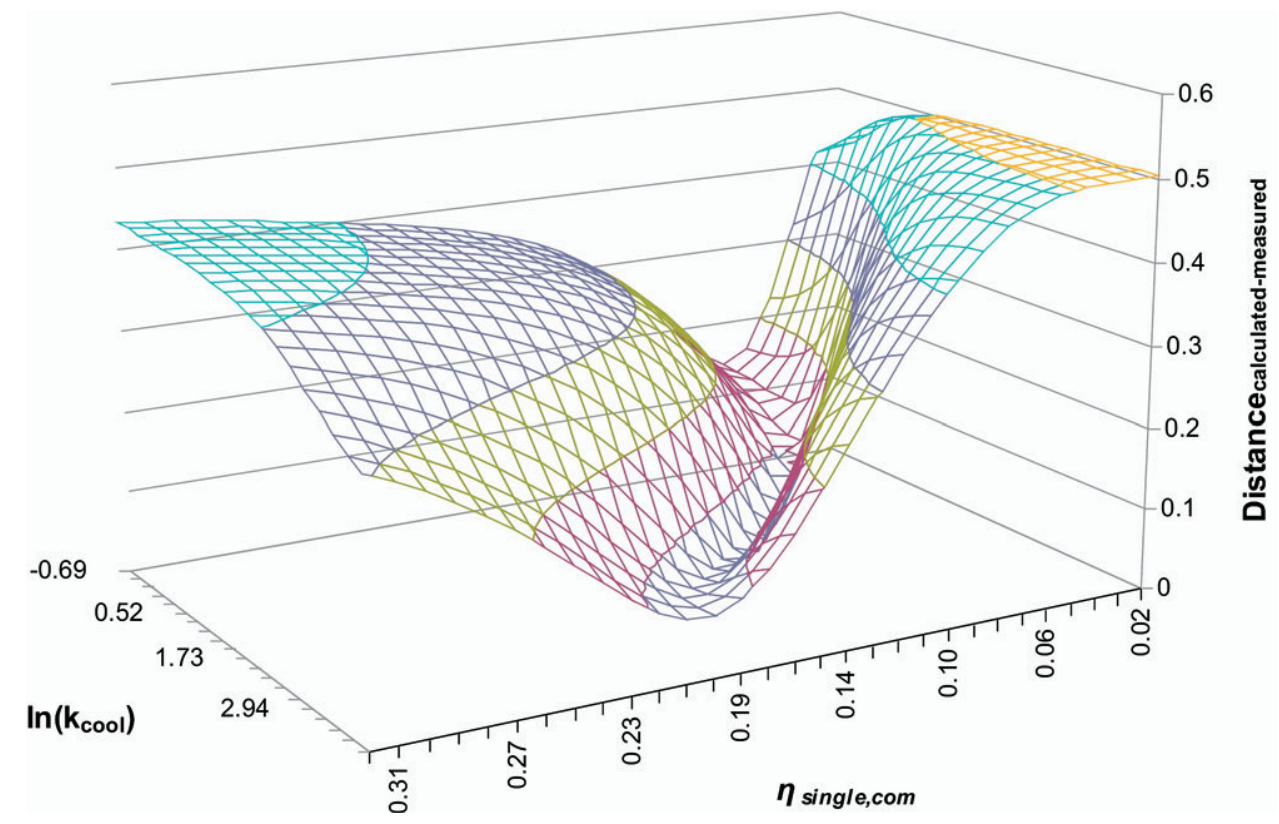

Figure 5. 3D plot of the overall fit (average distance between measured and calculated point) in the energy-transfer efficiency logarithm of IR cooling rate parameter space. 
data points were optimized using two parameters only increase confidence that the results are sound.

The results show collisional energy-transfer efficiency $\left(\eta_{\text {single,com }}\right)$ of $0.128 \pm 0.021$ and radiative cooling rate $\left(k_{\text {cool }}\right)$ of $7.5 \pm 0.5 \mathrm{~s}^{-1}$. Note that the latter means that ions are loosing half their internal energy in ca. 0.1 to $0.2 \mathrm{~s}$, so radiative cooling should be considered in all ion trapping experiments. The large uncertainty of the results mainly reflects strong interdependence of $\eta_{\text {single,com }}$ and $k_{\text {cool }}$, so underestimation of one could be compensated to a large degree by underestimating the other as well. The results also underline the importance of considering of various physicochemical processes simultaneously for modeling complex phenomena, such as mass spectrometric experiments.

\section{Acknowledgments}

The authors gratefully acknowledge financial support of OTKA T062727, Anyos Jedlik program of the Agency for Research Fund Management and Research Exploitation (Hungary). They are indebted to Ron Heeren for helpful discussions.

\section{References}

1. Marshall, A. G.; Hendrickson, C. L.; Jackson, G. S. Fourier Transform Ion Cyclotron Resonance Mass Spectrometry: A primer. Mass Spectrom. Rev. 1998, 17, 1-35.

2. Douglas, D. J.; Frank, A. J.; Mao, D. M. Linear Ion Traps in Mass Spectrometry. Mass Spectrom. Rev. 2005, 24, 1-29.

3. Beauchamp, J. L. Ion Cyclotron Resonance Spectroscopy. Ann. Rev. Phys. Chem. 1971, 22, 527.

4. Bogdanov, B.; Smith, R. D. Proteomics by FTICR Mass Spectrometry: Top Down and Bottom Up. Mass Spectrom. Rev. 2005, 24, 168-200.

5. Rompp, A.; Taban, I. M.; Mihalca, R.; Duursma, M. C.; Mize, T. H.; McDonnell, L. A.; Heeren, R. M. A. Examples of Fourier Transform Ion Cyclotron Resonance Mass Spectrometry Developments: From Ion Physics to Remote Access Biochemical Mass Spectrometry. Eur. J. Mass Spectrom. 2005, 11, 443-456.

6. Pitteri, S. J.; McLuckey, S. A. Recent Developments in the Ion/Ion Chemistry of High-Mass Multiply Charged Ions. Mass Spectrom. Rev. 2005, 24, 931-958.

7. Laskin, J.; Futrell, J. H. Activation of Large Ions in FT-ICR Mass Spectrometry. Mass Spectrom. Rev. 2005, 24, 135-167.

8. Heeren, R. M. A.; Vékey, K. Novel Method to Determine Collisional Energy Transfer Efficiency by Fourier Transform Ion Cyclotron Resonance Mass Spectrometry. Rapid Commun. Mass Spectrom. 1998, 12, $1175-1181$.

9. Guo, X. H.; Duursma, M. C.; Kistemaker, P. G.; Nibbering, N. M. M.; Vékey, K.; Drahos, L.; Heeren, R. M. A. Manipulating Internal Energy of Protonated Biomolecules in Electrospray Ionization Fourier Transform Ion Cyclotron Resonance Mass Spectrometry. J. Mass Spectrom. 2003, 38, 597-606.

10. Guo, X. H.; Duursma, M. C.; Al-Khalili, A.; Heeren, R. M. A. Experimental Calibration of the SORI-CID Internal Energy Scale: Energy Uptake and Loss. Int. J. Mass Spectrom. 2003, 225, 71-82.

11. Marshall, A. G.; Verdun, F. R. Fourier Transforms in NMR, Optical, and Mass Spectrometry, Elsevier Science: Amsterdam, 1990

12. Herrmann, K. A.; Somogyi, A.; Wysocki, V. H.; Drahos, L.; Vékey, K. Combination of Sustained Off-Resonance Irradiation and On-Resonance Excitation in FT-ICR. Anal. Chem. 2005, 77, 7626-7638.

13. Gauthier, J. W.; Trautman, T. R.; Jacobson, D. B. Sustained OffResonance Irradiation for Collision-Activated Dissociation Involving Fourier Transform Mass Spectrometry. Collision-Activated Dissociation Technique that Emulates Infrared Multiphoton Dissociation. Anal. Chim. Acta 1991, 246, 211-225.

14. Heck, A. J. R.; Dekoning, L. J.; Pinkse, F. A.; Nibbering, N. M. M. Mass-Specific Selection of Ions in Fourier-Transform Ion-Cyclotron Resonance Mass-Spectrometry-Unintentional Off-Resonance Cyclotron Excitation of Selected Ions. Rapid Commun. Mass Spectrom. 1991, 5, 406-414.
15. Cody, R. B.; Freiser, B. S. Collision-Induced Dissociation in a FourierTransform Mass-Spectrometer. Int. J. Mass Spectrom. Ion Processes 1982, 41, 199-204.

16. Cody, R. B.; Burnier, R. C.; Freiser, B. S. Collision-Induced Dissociation with Fourier-Transform Mass-Spectrometry. Anal. Chem. 1982, 54, $96-$ 101.

17. McLuckey, S. A.; Goeringer, D. E. Slow Heating Methods in Mass Spectrometry. J. Mass Spectrom. 1997, 32, 461-474.

18. Plass, W. R.; Cooks, R. G.; Goeringer, D. E.; McLuckey, S. A. Simulation of Ion Internal Energy Evolution During Collisional Processes in Quadrupole Ion Traps. J. Chem. Phys. 1996, 104, 2214-2215.

19. Fujiwara, M.; Naito, Y. Simulation for Internal Energy Deposition in Sustained Off-resonance Irradiation Collisional Activation Using a Monte Carlo Method. Rapid Commun. Mass Spectrom. 1999, 13, 1633 1638.

20. Laskin, J.; Byrd, M.; Futrell, J. Internal Energy Distributions Resulting from Sustained Off-Resonance Excitation in FTMS. I. Fragmentation of the Bromobenzene Radical Cation. Int. J. Mass Spectrom. 2000, 195/196, 285-302.

21. Laskin, J. Energetics and Dynamics of Peptide Fragmentation from Multiple-Collision Activation and Surface-Induced Dissociation Studies. Eur. J. Mass Spectrom. 2004, 10, 259-267.

22. Schnier, P. D.; Jurchen, J. C.; Williams, E. R. The Effective Temperature of Peptide Ions Dissociated by Sustained Off-Resonance Irradiation Collisional Activation in Fourier Transform Mass Spectrometry. J. Phys. Chem. B 1999, 103, 737-745.

23. Laskin, J.; Futrell, J. Internal Energy Distributions Resulting from Sustained Off-Resonance Excitation in Fourier Transform Ion Cyclotron Resonance Mass Spectrometry. II. Fragmentation of the 1-Bromonaphtalene Radical Cation. J. Phys. Chem. A 2000, 104, 5484-5494.

24. Dunbar, R. C.; McMahon, T. B. Activation of Unimolecular Reactions by Ambient Blackbody Radiation. Science 1998, 279, 194-197.

25. Dunbar, R. C. Infrared Radiative Cooling of Gas-Phase Ions. Mass Spectrom. Rev. 1992, 11, 309-339.

26. Dunbar, R. C. BIRD (blackbody infrared radiative dissociation): Evolution, Principles, and Applications. Mass Spectrom. Rev. 2004, 23, 127-158.

27. Guo, X. H.; Duursma, M.; Al-Khalili, A.; McDonnell, L. A.; Heeren, R. M. A. Design and Performance of a New FT-ICR Cell Operating at a Temperature Range of 77 to 438 K. Int. J. Mass Spectrom. 2004, 231, 37-45.

28. Drahos, L.; Vékey, K. MassKinetics: A Theoretical Model of Mass Spectra Incorporating Physical Processes, Reaction Kinetics, and Mathematical Descriptions. J. Mass Spectrom. 2001, 36, 237-263.

29. Drahos, L., Vékey, K. MassKinetics computer program, 2004; http:// www.chemres.hu/ms/masskinetics

30. Jockusch, R. A.; Paech, K.; Williams, E. R. Energetics from Slow Infrared Multiphoton Dissociation of Biomolecules. J. Phys. Chem. A 2000, 104, 3188-3196.

31. Becke, A. D. Density-Functional Exchange Energy Approximation with Correct Asymptotic Behavior. Phys. Rev. A 1988, 38, 3098-3100.

32. Becke, A. D. Density-Functional Thermochemistry. 2. The Effect of the Perdew-Wang Generalized Gradient Correlation Correction. J. Chem. Phys. 1992, 97, 9173-9177.

33. Lee, C.; Yang, W.; Parr, R. G. Development of the Colle-Salvetti Correlation Energy Formula into a Functional of the Electron Density. Phys. Rev. B 1988, 37, 785-789.

34. Meroueh, O.; Hase, W. L. Energy Transfer Pathways in the Collisional Activation of Peptides. Int. J. Mass Spectrom. 2000, 201, 233-244.

35. Wysocky, V. H.; Kenttamaa, H. I.; Cooks, R. G. Internal Energy Distributions of Isolated Ions After Activation by Various Methods. Int. J. Mass Spectrom. Ion Processes 1987, 75, 181.

36. Uggerud, E.; Derrick, P. J. Theory of Collisional Activation of Macromolecules. Impulsive Collisions of Organic Ions. J. Phys. Chem. 1991, 95, 1430-1436.

37. Bernshtein, V.; Oref, I.; Liu, C. L.; Hsu, H. C.; Ni, C. K. Experimental and Computational Investigation of Energy Transfer Between Azulene and Krypton. Chem. Phys. Lett. 2006, 429, 317-320.

38. Gorshkov, M. V.; Pasa-Tolic, L.; Smith, R. D. Pressure Limited Sustained Off-Resonance Irradiation for Collision-Activated Dissociation in Fourier Transform Mass Spectrometry. J. Am. Soc. Mass Spectrom. 1999, 10 15-18.

39. Muntean, F.; Armentrout, P. B. Guided Ion Beam Study of CollisionInduced Dissociation Dynamics: Integral and Differential Cross Sections. J. Chem. Phys. 2001, 115, 1213-1228.

40. Drahos, L.; Vékey, K. Entropy Evaluation Using the Kinetic Method: Is It Feasible? J. Mass Spectrom. 2003, 38, 1025-1042.

41. Gomory, A.; Vegh, P.; Sztaray, J.; Drahos, L.; Vékey, K. Kinetic Energy Release of Protonated Methanol Clusters Using the Low-Temperature Fast-Atom Bombardment: Experiment and Theory Combined. Eur. J. Mass Spectrom. 2004, 10, 213-220.

42. Pollreisz, F.; Gomory, A.; Sztaray, J.; Vegh, P.; Drahos, L.; Kiss, A.; Vékey, K. Very High Critical Energy Fragmentations Observed in CID. Int. J Mass Spectrom. 2005, 243, 41-47. 